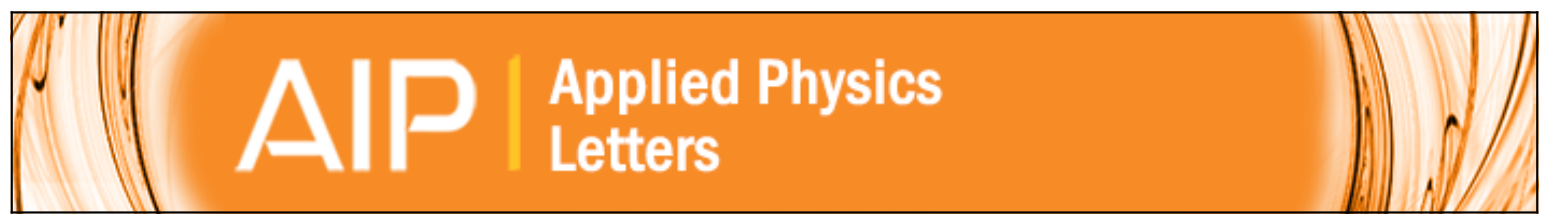

\title{
Surface plasmon enhanced responsivity in a waveguided germanium metal- semiconductor-metal photodetector
}

Fang-Fang Ren, Kah-Wee Ang, Junfeng Song, Qing Fang, Mingbin Yu, Guo-Qiang Lo, and Dim-Lee Kwong

Citation: Applied Physics Letters 97, 091102 (2010); doi: 10.1063/1.3485064

View online: http://dx.doi.org/10.1063/1.3485064

View Table of Contents: http://scitation.aip.org/content/aip/journal/apl/97/9?ver=pdfcov

Published by the AIP Publishing

\section{Articles you may be interested in}

Annealing effects of Au nanoparticles on the surface-plasmon enhanced p-Si/n-ZnO nanorods heterojunction photodetectors

J. Appl. Phys. 115, 173110 (2014); 10.1063/1.4875657

Metal-semiconductor-metal Ge photodetectors integrated in silicon waveguides

Appl. Phys. Lett. 92, 151114 (2008); 10.1063/1.2909590

Application of dopant segregation to metal-germanium-metal photodetectors and its dark current suppression mechanism

Appl. Phys. Lett. 92, 051110 (2008); 10.1063/1.2841061

Mixing characteristics of InGaAs metal-semiconductor-metal photodetectors with Schottky enhancement layers

Appl. Phys. Lett. 82, 3814 (2003); 10.1063/1.1579117

Hole Schottky barrier height enhancement and its application to metal-semiconductor-metal photodetectors J. Appl. Phys. 90, 5666 (2001); 10.1063/1.1415060

\section{A|P| Journal of}

Journal of Applied Physics is pleased to announce André Anders as its new Editor-in-Chief 


\title{
Surface plasmon enhanced responsivity in a waveguided germanium metal-semiconductor-metal photodetector
}

\author{
Fang-Fang Ren, ${ }^{a}$ Kah-Wee Ang, Junfeng Song, Qing Fang, Mingbin Yu, Guo-Qiang Lo, \\ and Dim-Lee Kwong \\ Institute of Microelectronics, Agency of Science and Technology Research (A*STAR), 11 Science Park Road, \\ Singapore Science Park II, Singapore 117685
}

(Received 11 June 2010; accepted 12 August 2010; published online 30 August 2010)

\begin{abstract}
The authors report on high transverse magnetic (TM)-mode responsivity in a waveguided germanium Schottky-barrier metal-semiconductor-metal photodetector on silicon-on-insulator substrate for operating wavelength at $1550 \mathrm{~nm}$. The employed aluminum interdigitated electrodes act as a one-dimensional rectangular grating above the depletion layer. By means of properly designed finger dimensions, surface plasmon polariton resonances can be excited at the interface of metal and silicon interfacial layer due to grating coupling. The resulting strong field intensities reach into active region, enabling high absorption under TM injection. At a voltage of $1 \mathrm{~V}$, the TM-mode photocurrent is measured over three times than that of transverse electric mode, in spite of the relatively larger TM insertion loss in the silicon waveguide. (c) 2010 American Institute of Physics. [doi:10.1063/1.3485064]
\end{abstract}

Surface plasmon polaritons (SPPs) are electromagnetic waves that are strongly coupled to free electron oscillation on the metal surface and propagate along the dielectric-metal interface. ${ }^{1}$ The presence of strong and localized optical intensities proved extremely efficient for semiconductor photodetectors in absorption enhancement and footprint shrinkage but most of design schemes focused on discrete components for operation with free-space illumination. ${ }^{2-4}$ In fact, an electrophotonic integrated circuit on a single chip platform requires waveguide-integrated photodetectors to receive optical signals through the input waveguides. So far, the research into this area is lagging behind.

In our previous work, germanium-on-silicon-oninsulator (Ge-on-SOI) photodetectors have been actively pursued owing to the large absorption coefficient and integration compatibility with silicon ( $\mathrm{Si}$ ) complementary metal-oxidesemiconductor (CMOS) process technology. ${ }^{5-8}$ For efficient coupling and propagating of transverse electric (TE)-mode light wave, our SOI substrate usually feature a Si core layer with very tight vertical confinement. A drawback found is the relatively lower transverse magnetic (TM) mode response, which mainly resulted from the larger TM insertion loss (including fiber-to-waveguide coupling loss and propagation loss).

In this work, we explore the use of SPPs in a waveguided Ge-on-SOI metal-semiconductor-metal (MSM) photodetector with interdigitated electrodes to achieve large TM enhancement at a standard communication wavelength of $1550 \mathrm{~nm}$. The plasmon-enhancement regime is realized by properly choosing the finger width and spacing in terms of the Bloch theorem and momentum conservation.

Figure 1(a) illustrates the cross-section structural geometry of our designed Ge-on-SOI MSM photodetector with an effective device width $W$ of $25 \mu \mathrm{m}$ and length $L$ of $50 \mu \mathrm{m}$. Figure 1(b) shows the top-view structure schematic of the interdigitated electrodes, which are defined by the figure width $w$ and spacing $s$ (or periodicity $a=w+s$ ). These elec-

\footnotetext{
a) Author to whom correspondence should be addressed. Electronic mail: fangfang.ren@gmail.com.
}

trodes effectively form a one-dimensional (1D) metallic rectangular grating with finite width, length, and height on the top surface of the massive multilayer. Taking into account the compatibility to Si-CMOS processes, the nontraditional plasmonic metal aluminum (Al) is employed in this work. A crystalline $\mathrm{Si}$ interfacial layer of $20 \mathrm{~nm}$ is designed to be inserted between the metal layer and the Ge region so as to increase the Schottky barrier and thus suppress the dark current of device. ${ }^{6}$ Figure $1(\mathrm{c})$ depicts a perspective view of our waveguide Si core layer with a thickness of $220 \mathrm{~nm}$ and a width of $500 \mathrm{~nm}$. The TE/TM-polarized (electric/magnetic field parallel to the $x$ axis) light wave propagates along the $z$-direction. When reaching the active regime, the $\mathrm{Si}$ waveguide width is adjusted with a taper to ensure efficient coupling of incidence photon from the routing $\mathrm{Si}$ waveguide up into the $\mathrm{Ge}$ region for absorption.

In our designed scheme, the SPP excitation is permitted by the counter-directional coupling between the guided mode propagating in the Si core layer and the copropagating SPP wave in the vicinity of $\mathrm{Al}-\mathrm{Si}$ interface with the assistance of Bragg grating. ${ }^{9,10}$ The first parameter to be chosen is the periodicity of the 1D Al grating, which can be roughly given by the momentum conservation law, ${ }^{11}$
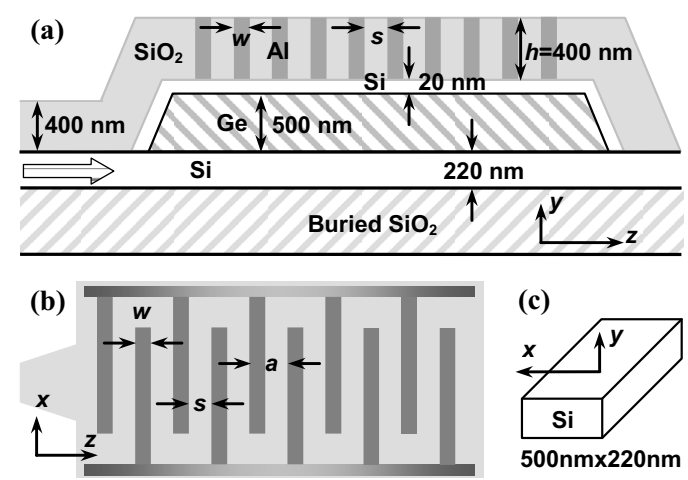

FIG. 1. Scheme of the Ge-on-SOI MSM photodetector with interdigitated electrodes. (a) Cross-sectional view of the device. (b) Top view of the interdigitated fingers. (c) Perspective view of the Si core layer with a thickness of $220 \mathrm{~nm}$ and a width of $500 \mathrm{~nm}$ where light propagates in the $z$-direction. 


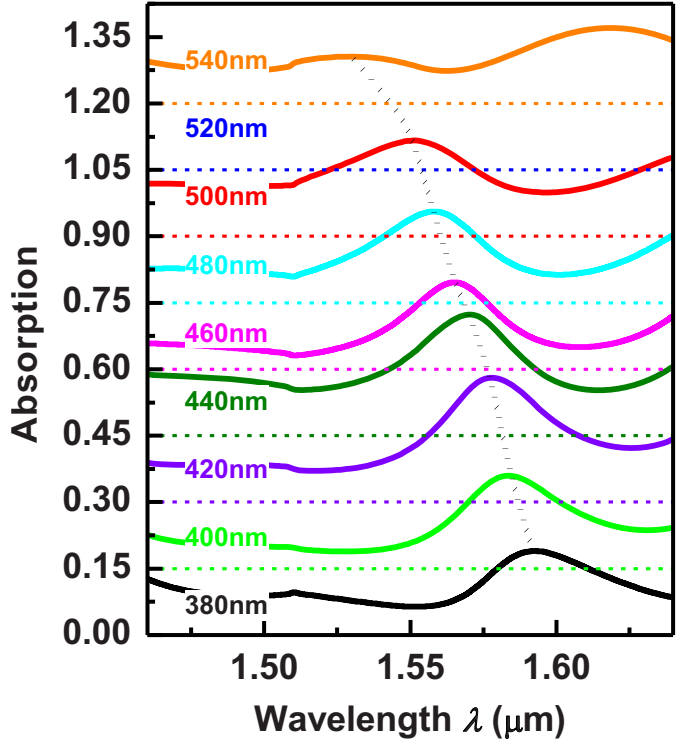

FIG. 2. (Color online) Calculated absorption spectra of the Ge region with grating periodicity $a=1500 \mathrm{~nm}$ and finger spacing $s$ varying from 380 to $540 \mathrm{~nm}$ under TM injection. The spectra are shifted upwards for clarity.

$$
k_{\mathrm{SPP}}=\beta \pm m G, \quad m=1,2,3, \ldots,
$$

where $m=1,2,3, \ldots$ and $G=2 \pi / a$ is the reciprocal vector of the periodic grating to facilitate additional wave vector for compensating the mismatching between the propagation constants of the core mode and SPP mode, respectively, i.e., $\beta$ $=(2 \pi / \lambda) n_{\text {core }}$ and $k_{\mathrm{SPP}}=(2 \pi / \lambda) n_{\mathrm{SPP}}$. Here $\lambda$ is the operating wavelength in vacuum. The core mode effective index, $n_{\text {core }}$, can be derived from BEAMPROP modeling, ${ }^{12}$ and the SPP effective index $n_{\mathrm{SPP}}$ approaches to $\sqrt{\varepsilon_{\mathrm{air}} \varepsilon_{\mathrm{Al}} /\left(\varepsilon_{\mathrm{air}}+\varepsilon_{\mathrm{Al}}\right)}$, where $\varepsilon_{\text {air }}$ and $\varepsilon_{\mathrm{Al}}$ are the frequency dependent dielectric constant of air and metal Al, respectively. When the wavelength $(\lambda)$ of injecting TM-mode light is $1550 \mathrm{~nm}$, the fundamental mode $n_{\text {core }}$ is calculated to be about 2.56 , and subsequently the periodicity $a$ is determined to be about $1500 \mathrm{~nm}$. With this value, the spectral range of the SPP resonance can be roughly controlled. Other parameters should be optimized by their individual variations. In Fig. 2, we numerically computed the absorption spectra of the Ge layer for the proposed device with the fixed periodicity of $1500 \mathrm{~nm}$ under TM injection. The SPP modes can be detected via the maximum points located slightly after Woods-Rayleigh anomaly modes. ${ }^{13,14}$ When the spacing $s$ is varied from 380 to $540 \mathrm{~nm}$, the peaks in absorbance display a gradual blueshift from 1592 to 1530 $\mathrm{nm}$. With $s=500 \mathrm{~nm}$, one SPP mode with resonance order of $m=1$ can be found at the communication wavelength of 1550 $\mathrm{nm}$. Thus, the theoretical modeling gives $a=1500 \mathrm{~nm}$ and $s=500 \mathrm{~nm}$ for the geometrical parameters of the $\mathrm{Al}$ grating.

Based on above design, we fabricated the plasmonic waveguided photodetector on an 8-in. SOI wafer with $2-\mu \mathrm{m}$-thick buried- $\mathrm{SiO}_{2}$. Figure 3 shows the top-view scanning electron microscopy (SEM) image of the device. The channel Si waveguide as described in Fig. 1(c) was first formed by dry etching. After active window definition, the pure Ge film with $500 \mathrm{~nm}$ thickness was selectively grown with a thin silicon-germanium buffer. ${ }^{6}$ The ultrathin crystalline $\mathrm{Si}$ layer was then deposited on the Ge region as a Schottky barrier. Subsequently, the $\mathrm{SiO}_{2}$ passivation layer of $4.00 \mathrm{~nm}$ was deposited and selectively dry etched to form

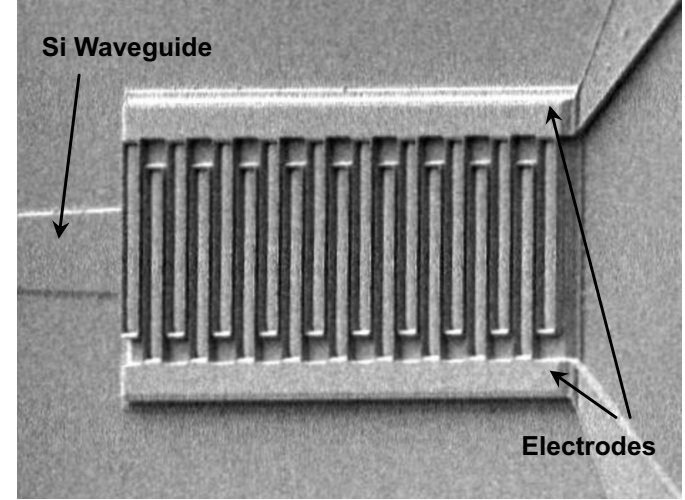

FIG. 3. Top-view SEM image of the Ge-on-SOI MSM photodetector featuring interdigitated electrodes with an effective device width $W$ of $25 \mu \mathrm{m}$ and length $L$ of $50 \mu \mathrm{m}$

contact hole. The metallization comprising of $\mathrm{Al}$ was deposited and patterned to complete the device fabrication.

The current-voltage characteristics of device illuminated under TE and TM modes are plotted in Fig. 4(a), as well as the dark currents for reference. At an applied bias of $1.0 \mathrm{~V}$, a low dark current of $\sim 0.5 \mu \mathrm{A}\left(0.4 \mathrm{nA} / \mu \mathrm{m}^{2}\right)$ was measured, which is below the typical $1.0 \mu \mathrm{A}$ generally considered to be the upper limit for high speed receiver design, consistent with the previous reported value on MSM devices. ${ }^{15}$ The optical measurement was performed by launching an incident electromagnetic (EM) wave with a photon wavelength of $1550 \mathrm{~nm}$ into the SOI waveguide and coupled into Ge active region for absorption. The effective incidence power into detector was determined by taking into account the coupling loss from fiber to waveguide and the waveguide propagation loss. In the case of TE illumination, the averaged insertion loss is measured to be about $4.5 \mathrm{~dB}$, including the fiber-to-waveguide coupling loss of $3.5 \pm 0.5 \mathrm{~dB}$ and propagation loss of $2.5 \pm 0.5 \mathrm{~dB} / \mathrm{cm}$ times 0.386 (the waveguide length in $\mathrm{cm}$ unit). While for TM mode, the measured coupling loss is larger as $4.5 \pm 0.5 \mathrm{~dB}$ and propagation loss as $3.5 \pm 0.5 \mathrm{~dB} / \mathrm{cm}$. Thus, the incidence light power reaching the $\mathrm{Ge}$ detector can be calculated to be $3 \mathrm{~mW}$ for TE mode and $1.5 \mathrm{~mW}$ for TM mode. At the applied bias of $1.0 \mathrm{~V}$, the photocurrents of the device are measured to be $0.527 \mathrm{~mA}$ and $1.622 \mathrm{~mA}$ under the individual TE and TM illumination, re
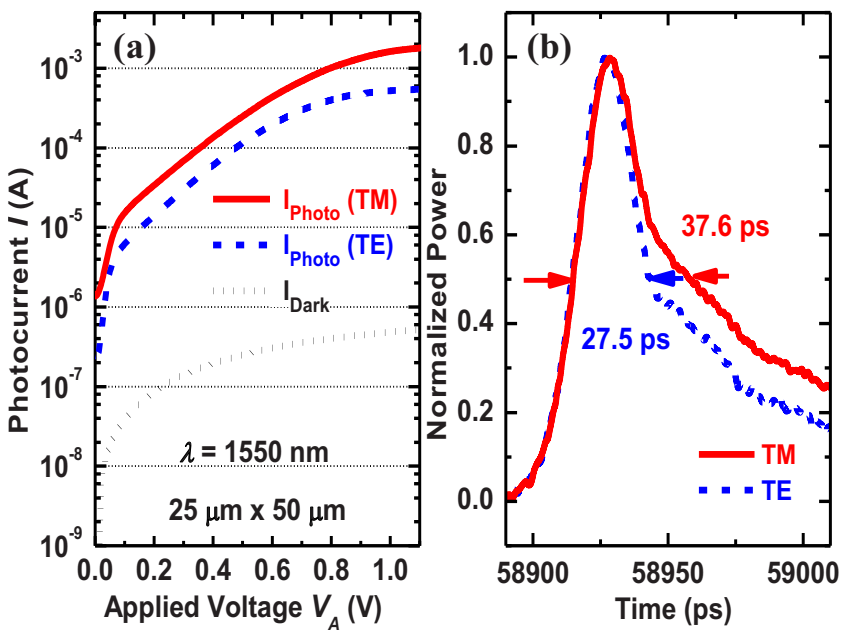

FIG. 4. (Color online) (a) Current-voltage characteristics and (b) normalized temporal response of the device with $T E$ and $T M$ injection, respectively. 


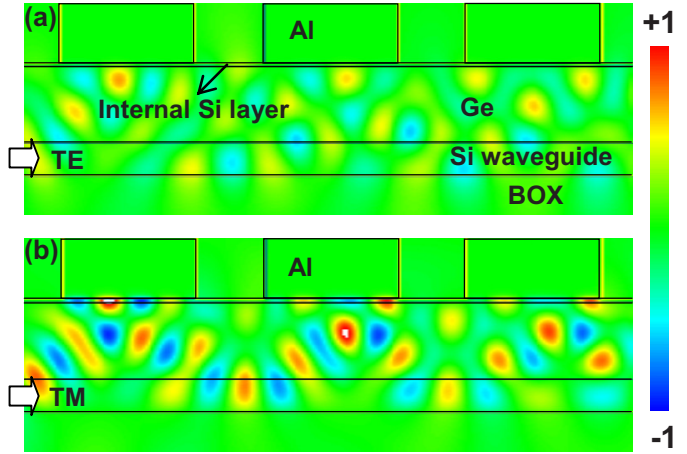

FIG. 5. (Color online) Distribution of normalized (a) electric field intensity $(E y)$ for TE mode and (b) magnetic field intensity $(H y)$ for TM mode in the device. The magnetic field is multiplied by free-space impedance for unification and comparison between electric and magnetic fields.

spectively, leading to responsivities of $0.176 \mathrm{~A} / \mathrm{W}$ and 1.081 $\mathrm{A} / \mathrm{W}$. The corresponding quantum efficiencies are thus calculated to be $14.1 \%$ and $86.7 \%$, respectively. It is worthy of note that responsivity and quantum efficiency under TM mode overwhelm that of TE mode, which is in contrary to the generally perceived trend where TE mode is dominant. ${ }^{5-8}$ We believe that the effect of the electrode grating should be responsible for the surprising results under TM illumination.

To analyze the physical mechanism associated with the SPP effect on the impressive enhancement, we employ the finite-difference time-domain method ${ }^{16}$ to perform the EM field distribution calculation for the carefully designed device by inputting a TE/TM-polarized fundamental waveguide mode with $\lambda=1550 \mathrm{~nm}$. The normalized electric/magnetic field intensities under TE/TM mode are plotted in Fig. 5. For unification and comparison, we multiplied the values of magnetic fields with free-space impedance $\sqrt{\mu_{0} / \varepsilon_{0}}$. The absorption of Ge layer is taken into account by setting the imaginary part of refractive index to be 0.00567 at $\lambda=1550 \mathrm{~nm} .^{17}$ For both TE and TM modes shown, the light waves are propagating inside the $\mathrm{Si}$ core layer and $\mathrm{Ge}$ region due to their higher refractive indices. Along the propagating direction ( $z$ axis), the optical intensity appears gradually weakening because of the Ge absorption and metal dissipation. By comparing Figs. 5(a) and 5(b), it is found that the maximal magnetic field density appears at the interface of $\mathrm{Al}$ and $\mathrm{Si}$ internal layer, which provides the evidence of interdigitated electrodes acting as a 1D grating coupler above the depletion layer. The distinct localization of optical energy below the electrodes is critically beneficial for the higher absorption, since it allows good overlap between optical energy density and electric fluxline. As such, the photon-generated electronhole pairs are separated quickly with high drift velocity, and the recombination possibility of electron and holes is reduced greatly. The final responsivity and quantum efficiency of device are thus increased.

Moreover, it is noted that the optical field under TMpolarization appears transverse oscillation in all the dielectric layers, not only locally concentrated in the vicinity of the metal layer boundaries as described for a SPP wave in general. It is because in our waveguide-integrated photodetector, the SPP mode is generated by counter-directional coupling between the SPP mode and the core guided mode. Such a coupled mode should thereby have hybrid nature in contrast to each of them. ${ }^{9,10}$ Owing to the strong field intensities ex- tending to the active layer thickness, the response of the detector could be further increased.

Besides the responsivity, the response speed is another critical performance parameter of detector, especially for applications such as optical communications and remote control. In this study, the frequency response of the MSM detector was obtained under incidence of a $1550 \mathrm{~nm}$ pulsed laser with 80 fs pulse width. Figure 4(b) shows the normalized temporal responses under TE/TM polarizations at an applied bias of $2.0 \mathrm{~V}$ were recorded by a high-speed oscilloscope. The full-width at half-maximum of the pulse response was measured as $\sim 27.5$ ps under TE mode, while a wider width of $\sim 37.6 \mathrm{ps}$ was obtained under TM mode. The corresponding $3 \mathrm{~dB}$ bandwidths reach $15.6 \mathrm{GHz}$ and $11.4 \mathrm{GHz}$, respectively. It is noted that the rise-time of response is almost same, and the major difference is the longer fall-time for TM illumination. It is easy to understand from the viewpoint of transport of photoexcited carriers. As discussed above, the strong localization of optical energy under TM excitation is found underneath at the interface of dielectric and metal, and thus the produced photoexcited electron-hole pairs are near the electrodes. The majority carriers (electrons) are easily collected by the nearest electrodes due to high drift velocity in Schottky-barrier induced build-in electric field. However, the minority carriers (holes) have to sweep through the whole finger spacing and be collected by the opposite electrode, leading to the long fall-time in Fig. 4(b). It indicates a trade-off that the enhancement in responsivity has to some extent to sacrifice the cost of reduced response speed.

The authors would like to thank Jian Wang for support in speed response measurements and Xiaoguang Tu for fruitful discussions. This work is supported by Agency for Science, Technology and Research (A*STAR) SERC (Science and Engineering Research Council) Grant Programme (SERC Grant No. 092154 0098), Singapore.

${ }^{1}$ H. Raether, Surface Plasmons (Springer, Berlin, 1988).

${ }^{2}$ J. Hetterich, G. Bastian, N. A. Gippius, S. G. Tikhodeev, G. von Plessen, and U. Lemmer, IEEE J. Quantum Electron. 43, 855 (2006).

${ }^{3}$ Z. Yu, G. Veronis, S. Fan, and M. L. Brongersma, Appl. Phys. Lett. 89, 151116 (2006).

${ }^{4}$ D. Okamoto, J. Fujikata, K. Nishi, and K. Ohashi, Jpn. J. Appl. Phys. 47, 2921 (2008)

${ }^{5}$ K. W. Ang, S. Y. Zhu, M. Yu, G. Q. Lo, and D. L. Kwong, IEEE Photonics Technol. Lett. 20, 754 (2008).

${ }^{6}$ K. W. Ang, S. Y. Zhu, J. Wang, K. T. Chua, M. B. Yu, G. Q. Lo, and D. L. Kwong, IEEE Electron Device Lett. 29, 704 (2008).

${ }^{7}$ J. Wang, W. Y. Loh, K. T. Chua, H. Zang, Y. Z. Xiong, T. H. Loh, M. B. Yu, S. J. Lee, G. Q. Lo, and D. L. Kwong, IEEE Electron Device Lett. 29, 445 (2008).

${ }^{8}$ J. Wang, W. Y. Loh, K. T. Chua, H. Zang, Y. Z. Xiong, S. M. F. Tan, M. B. Yu, S. J. Lee, G. Q. Lo, and D. L. Kwong, IEEE Photonics Technol. Lett. 20, 1485 (2008)

${ }^{9}$ G. Nemova and R. Kashyap, J. Opt. Soc. Am. B 24, 2696 (2007).

${ }^{10}$ G. Nemova and R. Kashyap, Opt. Commun. 281, 1522 (2008).

${ }^{11}$ Q. Liu and K. S. Chiang, Opt. Express 17, 7933 (2009).

${ }^{12}$ RSoft Design Group, BEAMPROP 8.2, http://www.rsoftdesign.com/.

${ }^{13}$ R. W. Woods, Phil. Mag. 4, 396 (1902).

${ }^{14}$ Y. W. Jiang, L. D. Tzuang, Y. H. Ye, Y. T. Wu, M. W. Tsai, C. Y. Chen, and S. C. Lee, Opt. Express 17, 2631 (2009).

${ }^{15}$ K. W. Ang, M. B. Yu, S. Y. Zhu, K. T. Chua, G. Q. Lo, and D. L. Kwong, IEEE Electron Device Lett. 29, 708 (2008).

${ }^{16} \mathrm{~A}$. Taflove, Computational Electrodynamics: The Finite-Difference TimeDomain Method, 2nd ed. (Artech House, Norwood, 2000).

${ }^{17}$ J. H. Weaver and H. P. R. Frederikse, CRC handbook of Chemistry and Physics (CRC, Boca Raton, 2001). 